\title{
Transfontanelläre Doppler-Sonographie der Hirnvenen im Säuglingsalter Teil II
} Pathologische Befunde

K.-H. Deeg
H. M. Lode

\author{
Trans-fontanellar Doppler Sonography of the Intracranial Veins in Infants \\ Part II - Pathology
}

\section{Zusammenfassung}

Mit der farbkodierten und gepulsten Doppler-Sonographie der intrakraniellen Venen können hämorrhagische Infarzierungen des Hirnparenchyms, Sinusvenenthrombosen und zerebrale arteriovenöse Malformationen diagnostiziert werden. Parenchymblutungen des Frühgeborenen kommen durch eine Kompression der Venae terminales und der subependymalen Venen zustande, die zu einer venösen Abflussstörung aus dem Marklager führt. Sie entsprechen somit pathologisch-anatomisch einem venösen Infarkt. Blutungen in die Basalganglien sind hochgradig verdächtig auf das Vorliegen einer Sinusvenenthrombose. Ein einseitiger Verschluss der V. cerebri interna führt zu einer einseitigen Blutung, ein beidseitiger Verschluss oder ein Verschluss der Vena Galeni magna oder des Sinus rectus hat eine beidseitige Basalganglienblutung sowie eine Ventrikeleinbruchsblutung und eine hämorrhagische Infarzierung des Hirnparenchyms zur Folge. Die häufigste arteriovenöse Malformation des Gehirns ist die AV-Malformation der Vena Galeni magna. Sonographisch imponiert sie als eine zystische Raumforderung hinter dem 3. Ventrikel, die sich farbdopplersonographisch als vaskuläre Fehlbildung darstellt. Mit der Doppler-Sonographie können die arteriellen Zuflüsse, meist die Aa. chorioideae posteriores und die venöse Drainage über den Sinus rectus, dargestellt werden.

\section{Schlliisselwörter}

Doppler-Sonographie · Hirnvenen · Säuglinge $\cdot$ hämorrhagische Infarkte · Sinusvenenthrombose · AV-Malformation Vena Galeni magna

\section{Abstract}

Colour coded Doppler sonography can be very helpful for the diagnosis of haemorrhagic infarction of the brain parenchyma, cerebral venous thrombosis and arterio-venous malformations of the brain. Intracranial haemorrhages into the brain parenchyma are caused by the compression of the subependymal and terminal veins which impede the venous drainage from the white matter. Haemorrhage of the basal ganglia is highly suspicious of cerebral venous thrombosis especially of the deep venous drainage. Unilateral occlusion of only one internal cerebral vein causes unilateral haemorrhage, bilateral thrombosis of both internal cerebral veins as well as occlusion of the great vein of Galens or the straight sinus causes bilateral haemorrhage of the basal ganglia as well as ventricular haemorrhage and haemorrhagic infarction of the white matter. The most common arterio-venous malformation of the brain is AV-malformation of Galens's vein. Sonographically, a pulsating cystic structure behind the 3rd ventricle can be shown. Colour coded Doppler sonography demonstrates the vascular nature of the cyst. Doppler sonography can show the feeding arteries, most frequently the posterior choroidal arteries and the venous drainage by the straight sinus.

\section{Key words}

Doppler sonography · cerebral veins · infants - haemorrhagic infarction - cerebral venous thrombosis $\cdot A V$-malformation of Galen's vein 
Mit der farbkodierten Doppler-Sonographie können die intrakraniellen Gefäße des Frühgeborenen, Neugeborenen und Säuglings durch die offene Fontanelle optimal abgebildet werden [12]. Neben der Darstellung der intrakraniellen Arterien können auch alle wichtigen intrakraniellen Venen mit der farbkodierten Doppler-Sonographie dargestellt und mit der gepulsten DopplerSonographie gemessen werden [12].

Neben der normalen Gefäßanatomie lassen sich wichtige Erkrankungen der venösen Drainage dopplersonographisch erfassen. Der Doppler-Sonographie kommt dabei einerseits bei der Diagnosestellung, andererseits bei der Verlaufskontrolle eine wichtige Rolle zu.

Anhand der hämorrhagischen Infarzierung des Hirnparenchyms, der Sinusvenenthrombose, des Infarktes der A. cerebri media und der AV-Malformation der Vena Galeni magna sollen die Möglichkeiten der Doppler-Sonographie des intrakraniellen Venensystems im Säuglingsalter dargestellt werden.

\section{Hämorrhagische Infarzierung des Hirnparenchyms beim Frühgeborenen}

Hirnblutungen des Frühgeborenen können in Abhängigkeit vom Schweregrad klassifiziert werden. Die initiale Graduierung nach Papile erfolgte in vier Schweregrade, wobei Grad-IV-Blutungen Parenchymblutungen darstellten [19]. Grad-IV-Blutungen wurden dabei als Ausdehnung von schweren Ventrikeleinbruchsblutungen ins Hirnparenchym angesehen [19]. Nach Volpe handelt es sich bei Grad-IV-Blutungen jedoch nicht einfach um eine Ausdehnung einer ausgeprägten Ventrikelblutung ins Parenchym, sondern um einen venösen Infarkt, sodass Grad-IV-Blutungen in seiner Klassifikation der Blutungen des Frühgeborenen nicht enthalten sind [23]. Das hat die pädiatrische Sektion der DEGUM im Jahre 1998 in einer neuen Klassifikation der Hirnblutungen (Grad I bis III) des Frühgeborenen berücksichtigt, in der Parenchymblutungen als eigene Entität aufgefasst werden [11]. Mit der farbkodierten Doppler-Sonographie konnte Taylor 1995 zeigen, dass die Vermutung von Volpe richtig war und es sich bei Grad-IVBlutungen um hämorrhagische Infarkte handelt [21].

Blutkoagel im Seitenventrikel (Grad-III-Blutungen) sowie große subependymale Blutungen können die Venae terminales am Boden der Seitenventrikel und die subependymalen Venen am Dach der Seitenventrikel komprimieren und den Abfluss aus den medullären Venen behindern (Abb.1a, b). Als Folge der Abflussstörung über die Venae terminales kann es zur hämorrhagischen Infarzierung des Hirnparenchyms kommen (Abb. 2, 3) [21, 23].

Eine ausgeprägte Ventrikeleinbruchsblutung (Grad III) führt zunächst nur zur Verlagerung der Venae terminales am Boden der Seitenventrikel (Abb.2a). Dabei kommt es mit zunehmender Kompression der Venen nach den Bernoulli-Gesetzen zu einem Anstieg der Flussgeschwindigkeiten (Abb. 2b). Der Abfluss aus den medullären Venen kann dabei in zunehmendem Maße behindert werden, sodass die Blutströmung letztendlich sistiert und
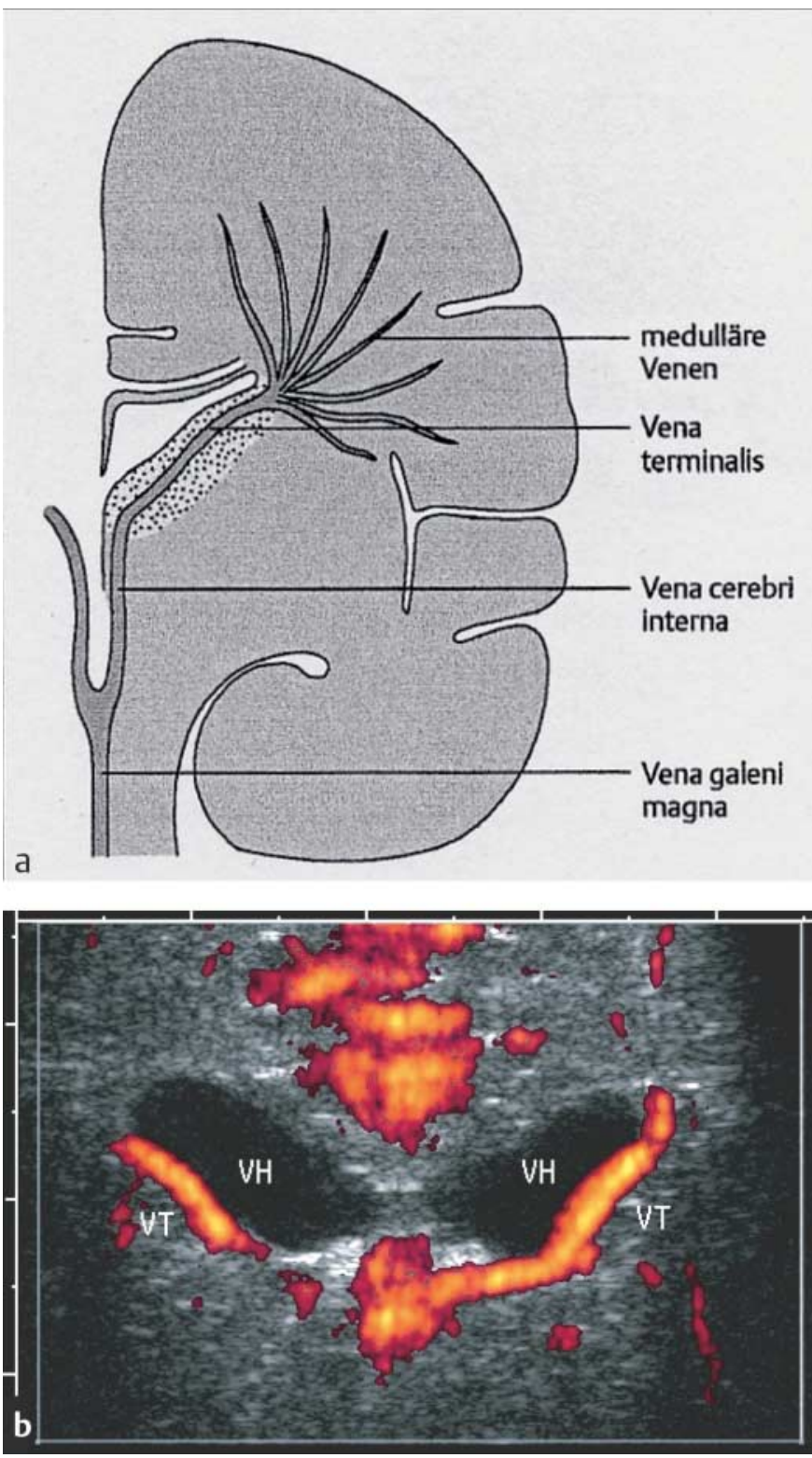

Abb. 1 Genese der hämorrhagischen Infarzierung des Hirnparenchyms. a Schematische Darstellung der venösen Drainage des Marks. Die Venae terminales drainieren das Blut der medullären Venen. Intraventrikuläre Blutungen können zu einer Kompression der Vena terminalis führen und eine Abflussstörung und hämorrhagische Infarzierung des Hirnparenchyms zur Folge haben. b Darstellung der Venae terminales am Boden der Seitenventrikel mit dem Power-Doppler. VH = Vorderhorn des Seitenventrikels; VT = Vena terminalis.

Fig. 1 Genesis of hemorrhagic infarction of the brain in premature infants. a Diagramme of the venous drainage of the white mater. The terminal veins drain the blood from the medullary veins. Blood clots within the ventricular system may compress the terminal veins, impede the venous drainage and cause hemorrhagic infarction of the brain parenchyma. b Power Doppler sonography of the terminal veins at the bottom of the lateral ventricles. $\mathrm{VH}=$ frontal horn of the lateral ventricle; $\mathrm{VT}=$ terminal vein . 

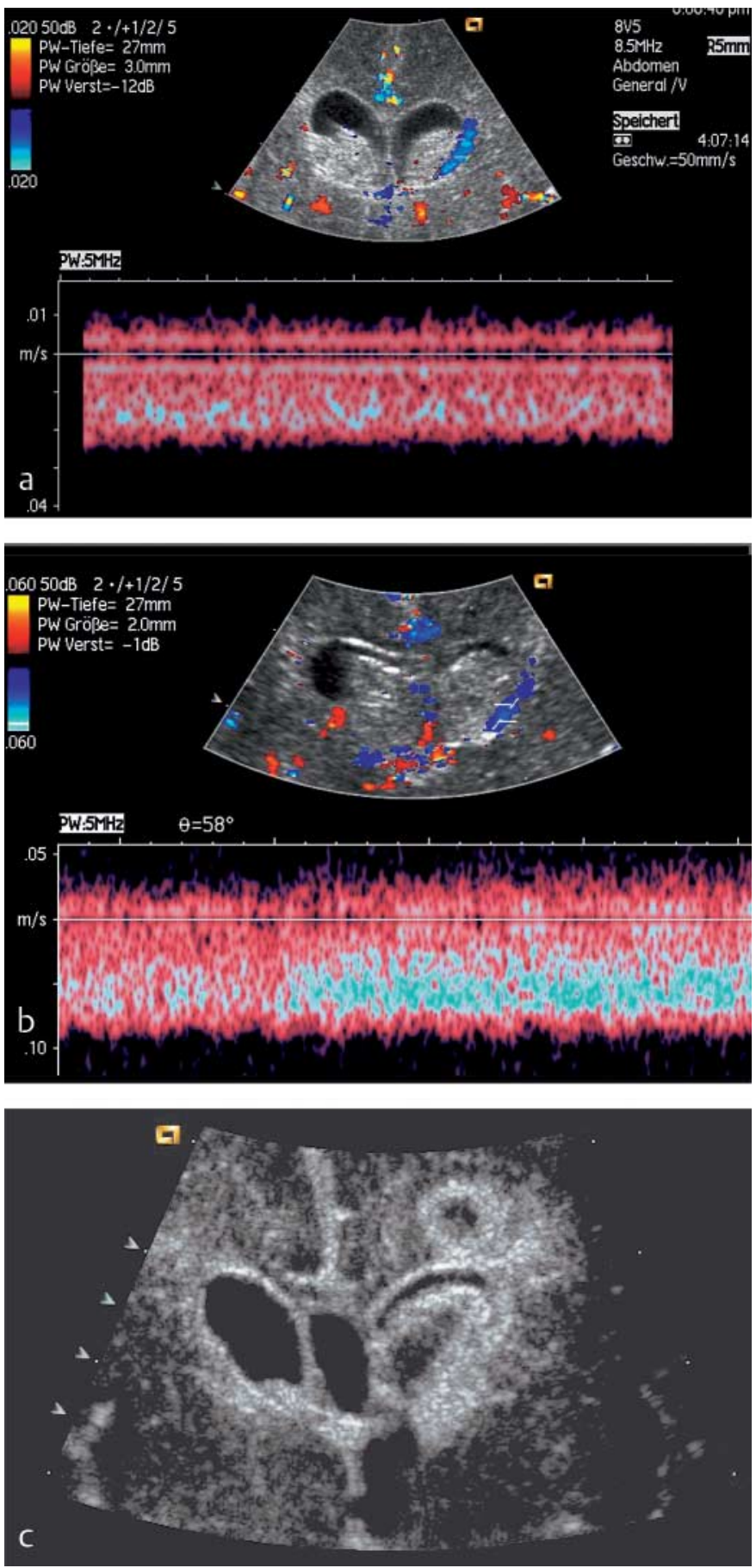

eine hämorrhagische Infarzierung des Hirnparenchyms resultiert (Abb. 2c und 3a, b) [22].

Nach eingetretener hämorrhagischer Infarzierung des Hirnparenchyms kann in der Vena terminalis keine Blutströmung mehr nachgewiesen werden [22]. Umgekehrt konnte Veyrac bei keinem Kind mit nachweisbarem Fluss in den Venae terminales eine hämorrhagische Infarzierung des Hirnparenchyms finden [22].

Mit der farbkodierten gepulsten Doppler-Sonographie konnte somit die Genese der Parenchymblutungen des Frühgeborenen als venöser Infarkt eindeutig bewiesen werden.
Abb. 2 Entwicklung einer hämorrhagischen Infarzierung des Hirnparenchyms. a Mäßiggradige Ventrikeleinbruchsblutung (Grad II), die zu einer leichten Verlagerung der Vena terminalis geführt hat. Normale Flussgeschwindigkeit mit $2,5 \mathrm{~cm} / \mathrm{s}$. b Zunahme der Ventrikelblutung (Grad III). Dadurch kommt es zu einer deutlichen Verlagerung der Vena terminalis, sodass das Gefäß komprimiert wird und die Flussgeschwindigkeit auf $10 \mathrm{~cm} / \mathrm{s}$ ansteigt. Im weiteren Verlauf kann es zu einer weiteren Kompression des Hirngefäßes kommen, sodass sich eine hämorrhagische Infarzierung des Hirnparenchyms entwickelt. c Hämorrhagische Infarzierung des Hirnparenchyms durch Kompression der Vena terminalis nach schwerer Ventrikeleinbruchsblutung (Koronarschnitt). Die Abbildung zeigt neben einem hämorrhagischen Infarkt große Blutkoagel im Seitenventrikel auf der rechten Bildseite. Fig. 2 Development of hemorrhagic infarction of the brain parenchyma. a Moderate ventricular hemorrhage (Grade II) causes minimal displacement and compression of the terminal vein. Normal flow velocity of $2.5 \mathrm{~cm} / \mathrm{s}$. b Increase of the intraventricular hemorrhage (Grade III) causes moderate displacement and additional compression of the vein followed by an increase of the flow velocity to $10 \mathrm{~cm} / \mathrm{s}$. In the further course the flow velocity may further increase causing hemorrhagic infarction of the brain parenchyma. $\mathbf{c}$ Hemorrhagic infarction of the brain parenchyma caused by compression of the terminal veins by severe intraventricular hemorrhage (coronal section). Beside hemorrhagic infarction of the brain parenchyma the lateral ventricle is completely filled with blood clots.

Thrombosen großer und/oder kleiner Hirnvenen wurden früher fast ausschließlich mit der Angiographie, in den letzten Jahren vorwiegend computertomographisch und/oder kernspintomographisch diagnostiziert. Mithilfe der hochauflösenden Farb-DuplexSonographie konnten im letzten Jahrzehnt einige Autoren die Diagnose einer Thrombose der Hirnvenen auch sonographisch stellen $[4,7,13,16]$. Voraussetzung ist eine exakte Kenntnis des normalen Verlaufs der großen und kleinen Hirnvenen sowie deren Darstellungsmöglichkeiten [12]. 

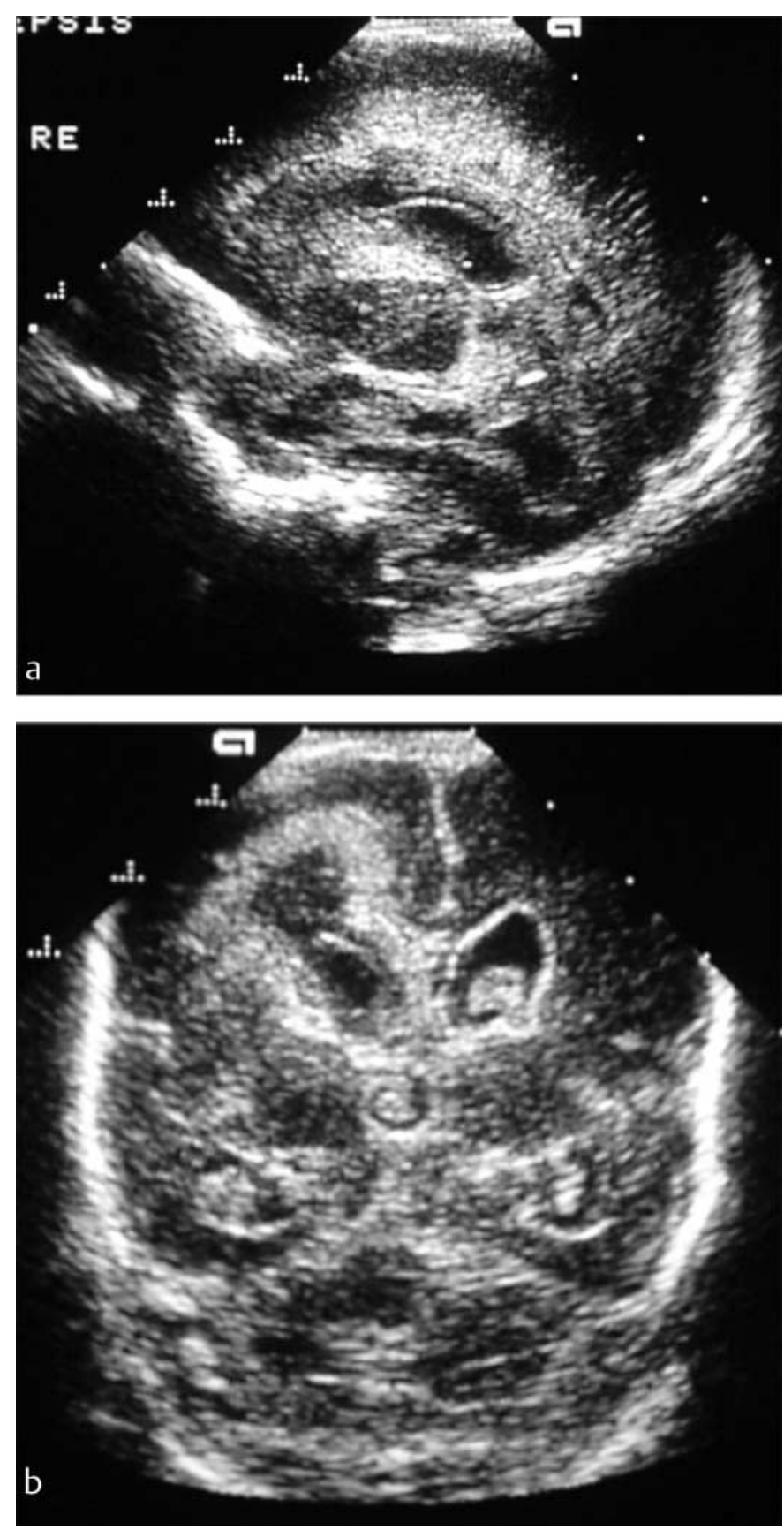

Abb. 3 Hämorrhagische Infarzierung des Hirnparenchyms als Folge einer kompletten Verlegung des venösen Abstroms aus der Vena terminalis rechts. a Parasagittalschnitt durch den Seitenventrikel, b Koronarschnitt.

Fig. 3 Hemorrhagic infarction of the brain parenchyma as a result of complete obstruction of the venous outflow by the right terminal vein. a Parasagittal section through the lateral ventricle, $\mathbf{b}$ coronal section.

Beim Neugeborenen kann der venöse Rückfluss in den Hirnvenen von einer Vielzahl von Faktoren beeinflusst werden, die zu einer Hirnvenenthrombose führen können. Die wichtigsten Risikofaktoren für eine Thrombose der Hirnvenen sind angeborene oder erworbene Thrombophilien (Tab.1), die Hämokonzentration und Polyzytämie, Infektionen, insbesondere Sepsen, die perinatale Asphyxie sowie akute Dehydratationen und angeborene Herzfehler $[1-3,7]$.
Tab. 1 Thrombophilieabklärung bei Hirnvenenthrombosen

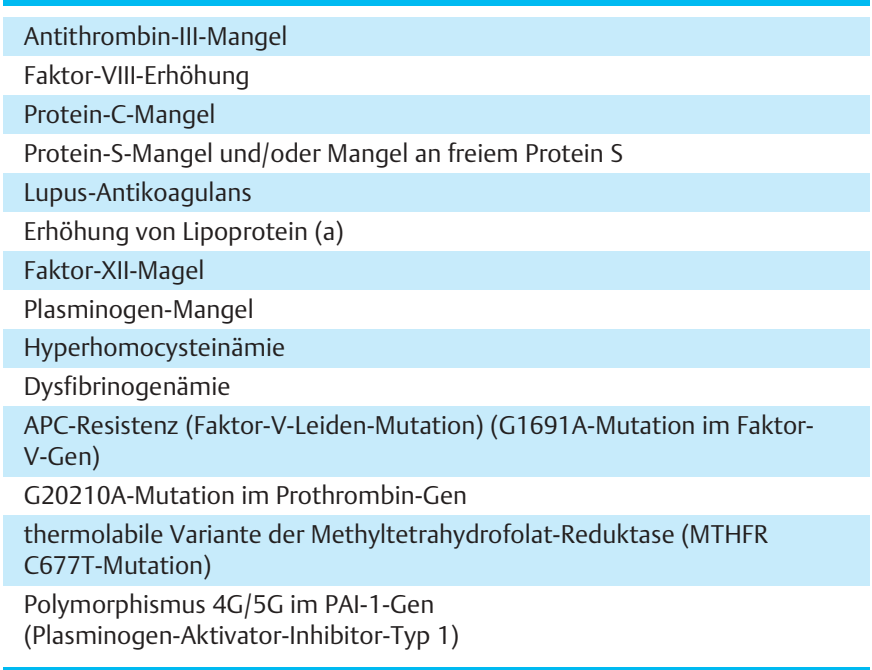

Beim Frühgeborenen mit Atemnotsyndrom und mechanischer Beatmung kann ein erhöhter intrathorakaler Druck den venösen Rückfluss behindern. Weiterhin können Blutdruckschwankungen, posthämorrhagische Hypovolämien sowie ein offener Ductus arteriosus Botalli den venösen Rückfluss aus dem Gehirn negativ beeinflussen.

Seltene Ursachen von Hirnvenenthrombosen sind das nephrotische Syndrom, Gerinnungsstörungen bei Antithrombin-III- und Protein-C-Mangel.

Trotz der mannigfaltigen Risikofaktoren, die zur Hirnvenenthrombose prädisponieren, bleiben ca. 25\% der Sinusvenenthrombosen ungeklärt [1].

Klinische Symptome, die auf eine Hirnvenenthrombose hindeuten, sind zerebrale Krampfanfälle, ein meist herabgesetzter Muskeltonus und Lethargie. Das mit Abstand häufigste Symptom sind jedoch zerebrale Krampfanfälle, die bei ca. $2 / 3$ aller Kinder mit Hirnvenenthrombose auftreten [7, 23].

Die Diagnose einer Hirnvenenthrombose kann farbdopplersonographisch erfolgen $[4,9,13,16]$. Hierzu müssen alle großen venösen Sinus (Sinus sagittalis superior, inferior und transversus) sowie die inneren Hirnvenen (Venae cerebri internae, Vena Galeni magna und Sinus rectus) in Sagittal- und Koronarschnitten dargestellt werden $[7,12]$.

Am häufigsten ist der Sinus sagittalis betroffen. Im zweidimensionalen Schnittbild stellt sich der Sinus sagittalis im Falle einer Sinusvenenthrombose als dreieckige, echogene Struktur mit konvexer Begrenzung dar (Abb.4a). Im Normalfall imponiert der Sinus sagittalis als echofreie dreieckige Struktur mit konkaver Begrenzung [12]. Beim Schreien nimmt der Sinus an Größe zu. Im Falle einer Sinusvenenthrombose unterbleibt die Größenänderung [7]. Mithilfe der farbkodierten und gepulsten Doppler-Sonographie kann eine Sinusvenenthrombose sicher nachgewiesen oder ausgeschlossen werden [4, 9]. Im Falle einer Sinusvenen- 

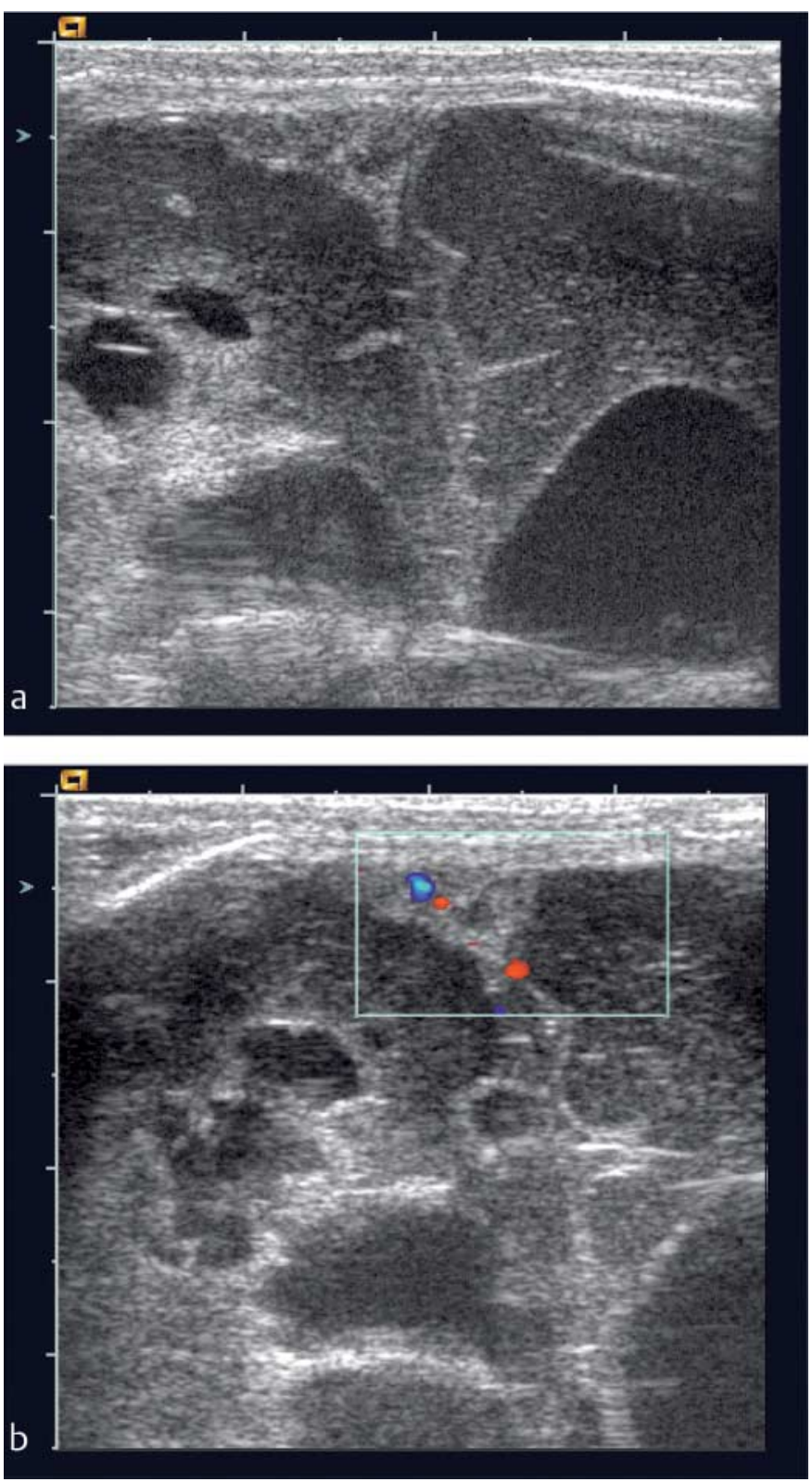

Abb. 4 Sinusvenenthrombose des Sinus sagittalis superior bei einem Frühgeborenen der 31. Schwangerschaftswoche mit posthämorrhagischem Hydrozephalus. Koronare Ausschnittsvergrößerung der Hirnoberfläche. a Zweidimensionales Schnittbild: echogene Darstellung des Sinus sagittalis superior mit konvexer Begrenzung. b Farbkodierte Doppler-Sonographie: fehlende Blutströmung im Sinus sagittalis superior.

Fig. 4 Cerebral venous thrombosis of the superior sagittal sinus in a prematurely born infant of 31 weeks of gestation with posthemorrhagic hydrocephalus. High resolution coronal scan of the brain suface. a 2-dimensional image: The superior sagittal sinus is displayed echogenic with convex borders. b Colour coded Doppler sonography shows no blood flow within the sinus.

thrombose lässt sich mit beiden Methoden keine Blutströmung finden (Abb. 4b).

Nach Volpe [23] betreffen ca. 85\% aller Hirnvenenthrombosen den Sinus sagittalis superior. Meist breitet sich die Thrombose vom Sinus sagittalis superior auf den Sinus transversus und rectus aus, die im nach hinten geneigten Koronarschnitt (Sinus transversus) und im medianen Sagittalschnitt (Sinus rectus) dargestellt werden können.

In seltenen Fällen geht die Thrombose von den inneren Hirnvenen (V. cerebri internae und Vena Galeni magna) aus. Alle drei Venen können mit der farbkodierten und gepulsten Doppler-Sonographie dargestellt werden. Die Differenzierung der beiden Venae cerebri internae gelingt im nach hinten geneigten Koronarschnitt, während die Vena Galeni magna am besten im medianen Sagittalschnitt abgebildet und gemessen wird [12].

Im weiteren Verlauf kann es in Abhängigkeit vom Ort des venösen Verschlusses zu unterschiedlichen Folgeschäden kommen.

Neben ischämischen Läsionen können auch hämorrhagische Schädigungsmuster auftreten:

- Im Falle eines Verschlusses des Sinus sagittalis superior kommt es zu bilateralen, v.a. frontal und paramedian lokalisierten, subkortialen hämorrhagischen Infarkten [7].

- Seltener ist die tiefe weiße Substanz betroffen.

- Bei Verschluss der inneren Venen resultiert ein ein- oder beidseitiger hämorrhagischer Infarkt des Thalamus, der angrenzenden Basalganglien sowie der Capsula interna (Abb.5 und 8). Weiterhin kann es zur Ventrikeleinbruchsblutung und zur hämorrhagischen Schädigung der weißen Substanz kommen.

Beim Nachweis einer unilateralen oder bilateralen Echogenitätsvermehrung im Bereich der Basalganglien liegt bis zum Beweis des Gegenteils immer eine Hirnvenenthrombose vor (Abb. 5a, b).

Bei Diagnose einer Blutung in die Basalganglien muss deswegen immer eine subtile dopplersonographische Darstellung aller Hirnvenen erfolgen $[7,12)$. Liegt gleichzeitig eine Ventrikeleinbruchsblutung und eine Schädigung des Marklagers der Großhirn-Hemisphären vor (Abb. 5 und 8), muss eine Obstruktion im Bereich der inneren zerebralen Venen (Venae cerebri interna, Vena Galeni magna und/oder Sinus rectus) ausgeschlossen werden.

Abb. 5 zeigt eine bilaterale Blutung in die Basalganglien und Capsula interna sowie eine beidseitige Ventrikeleinbruchsblutung und eine gleichzeitige Schädigung der weißen Substanz.

Die dopplersonographische Flussmessung in Abb. 6a zeigte keine Blutströmung im Sinus sagittalis superior. Der venöse Abstrom erfolgte über medulläre Venen zum Sinus sagittalis inferior (Abb. 6a).

- Die farbkodierte Duplex-Sonographie eignet sich einerseits zur Diagnosestellung einer Hirnvenenthrombose, andererseits v. a. für Verlaufskontrollen. Neben der Rekanalisierung können Umgehungskreisläufe nachgewiesen werden.

- In jedem Fall sollte jedoch bei sonographischer Diagnose einer Hirnvenenthrombose eine MR-Angiographie durchgeführt werden, um das wahre Ausmaß der Thrombose zu erfassen. Für den weiteren Verlauf sind sonographische Kontrollen ausreichend.

Eine Blockade der inneren zerebralen Venen führt über die venöse Abflussstörung zu folgenden Schädigungen: 

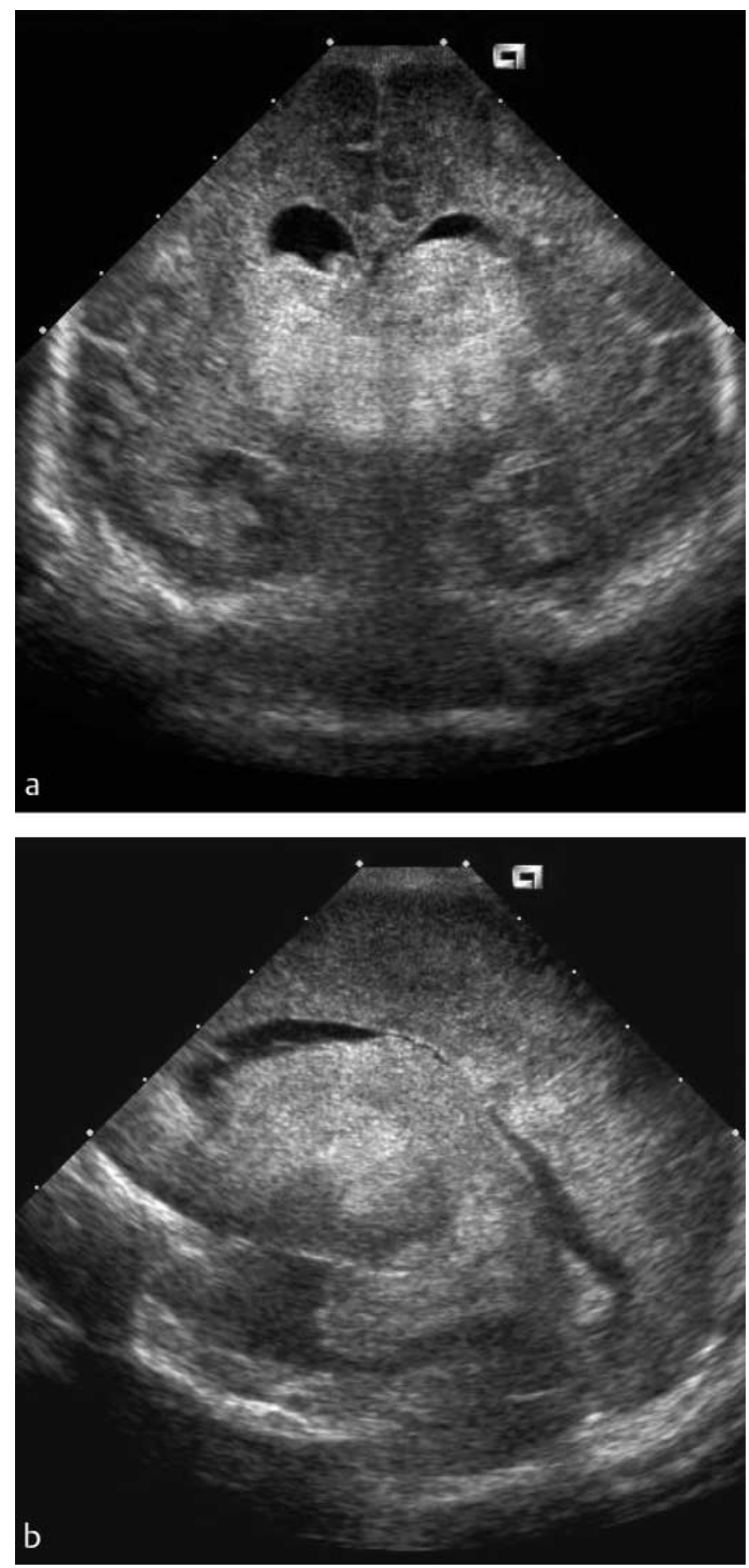

Abb. 5 Bilateraler hämorrhagischer Infarkt der Basalganglien und Ventrikeleinbruchsblutung bei einem Frühgeborenen von drei Wochen. Verdacht auf Hirnvenenthrombose. a Koronarschnitt: Echogenitätsvermehrungen in beiden Seitenventrikeln und im Bereich der Basalganglien. b Sagittalschnitt: Echogenitätsvermehrungen im Bereich der Seitenventrikel im Sinne einer Ventrikeleinbruchsblutung und im Bereich der Basalganglien im Sinne eines hämorrhagischen Infarkts.

Fig. 5 Bilateral hemorrhagic infarction of the basal ganglia and additional intraventricular hemorrhage in a prematurely born infant, suspicious of cerebral venous thrombosis. a Coronal section: Increased echogenicity in both lateral ventricles and basal ganglia. b Sagittal section: Increased echogenicity within the lateral ventricle (intraventricular hemorrhage) and in the region of the basal ganglia (hemorrhagic infarction).
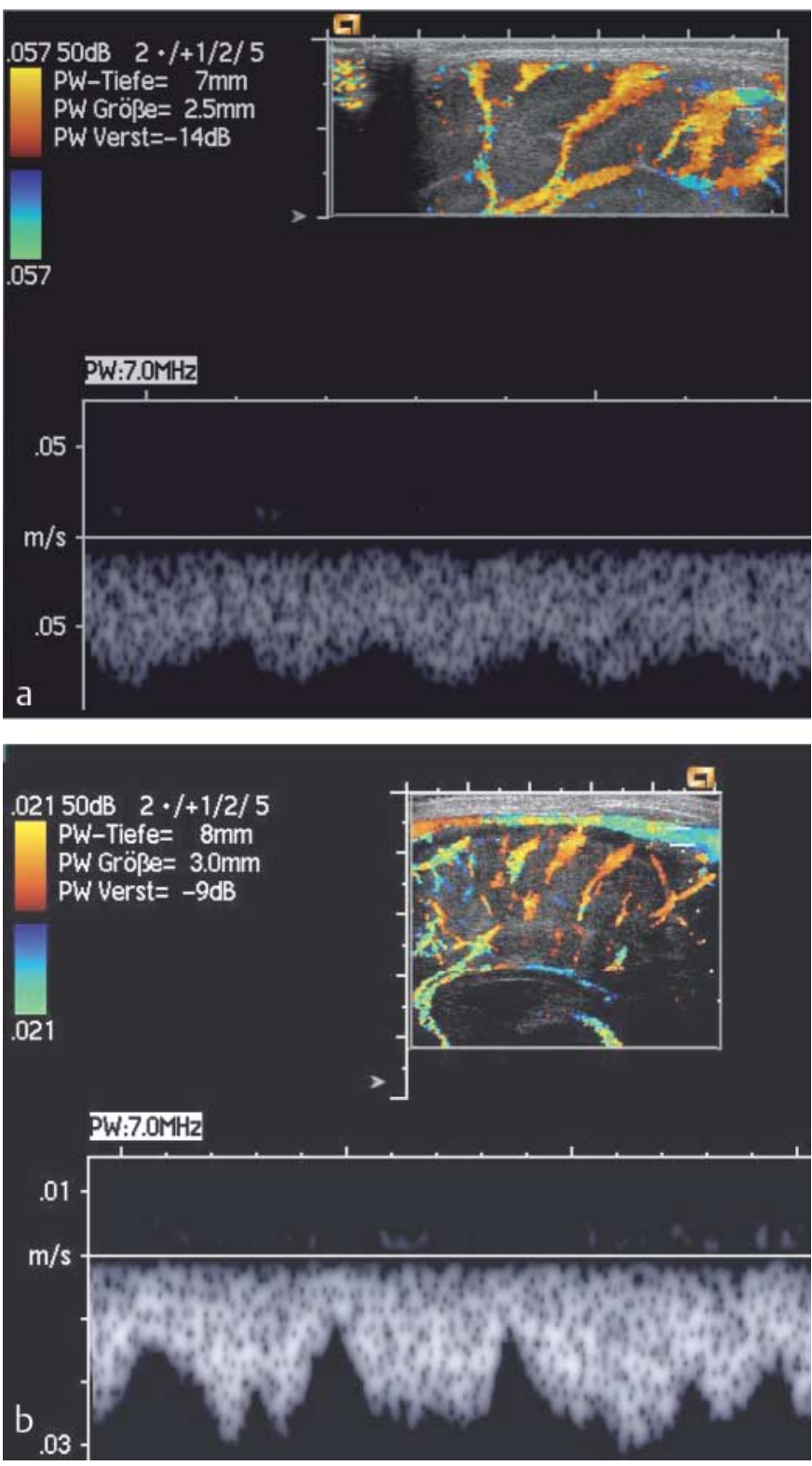

Abb. 6 Sinusvenenthrombose des Sinus sagittalis superior. Vergleich zwischen der Patientin a und dem gesunden Zwillingsbruder b. a Patientin: Sinus sagittalis superior mit der farbkodierten Doppler-Sonographie nicht darstellbar. Abfluss über transzerebrale Kollateralgefäße zum Sinus sagittalis inferior (negativer Fluss; normalerweise positiver Fluss). b Gesunder Bruder: Sinus sagittalis superior durchgängig, normale Blutströmung im Sinus sagittalis superior.

Fig. 6 Thrombosis of the superior sagittal sinus. Comparison between the female patient $\mathbf{a}$ and the healthy brother $\mathbf{b}$. a Female Patient: Colour coded Doppler sonography shows no flow within the superior sagittal sinus. Venous drainage by the way of transcerebral collaterals to the inferior sagittal sinus (negative flow in comparison to the positive flow in healthy individuals). b Healthy brother: Superior sagittal sinus patent. Pulsed Doppler Sonography shows normal blood flow within the superior sagittal sinus. 


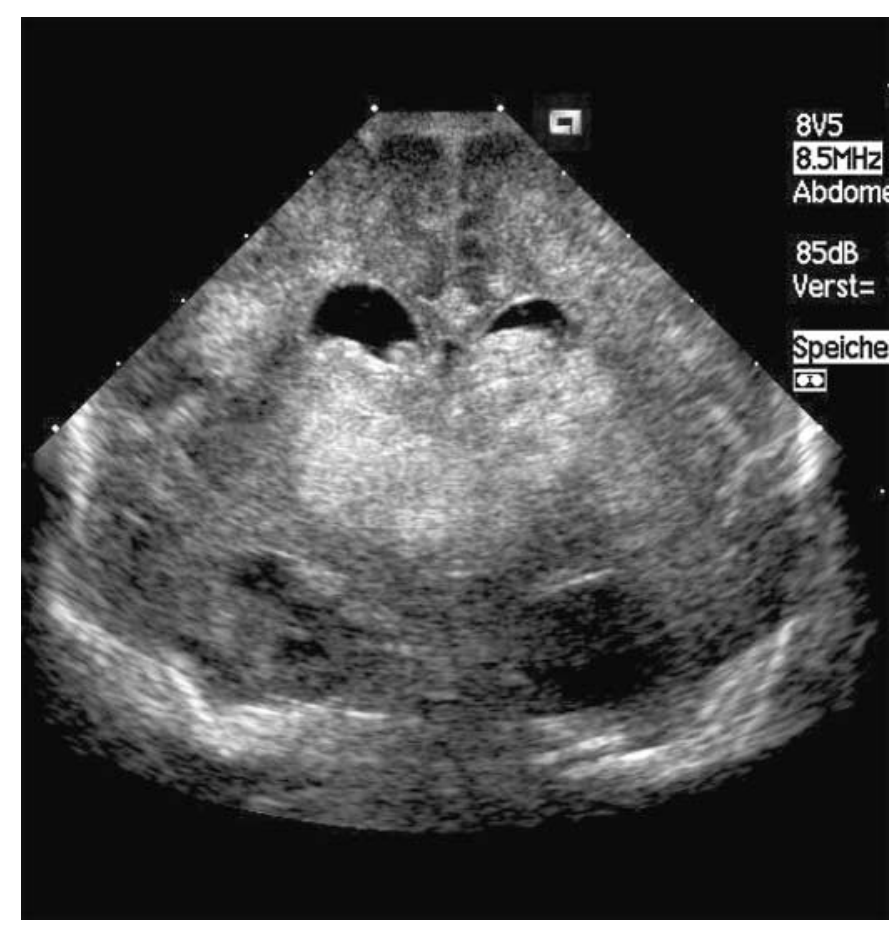

Abb. 7 Konsequenzen bei Sinusvenenthrombose und Verschluss der Venae cerebri internae: Verschluss der Venae terminales: $\rightarrow$ hämorrhagische Infarzierung der weißen Substanz; Verschluss der Venae chorioideae: $\rightarrow$ Ventrikelblutung; Verschluss der Venae thalamostriatae: $\rightarrow$ hämorrhagische Infarzierung der Basalganglien.

Fig. 7 Consequences of cerebral venous thrombosis: occlusion of the terminal veins: $\rightarrow$ hemorrhagic infarction of the white matter; occlusion of the choroideal veins: $\rightarrow$ intraventricular hemorrhage; occlusion of the lenticulo striatic veins: $\rightarrow$ hemorrhagic infarction of the basal ganglia.

1. Der Verschluss der Venae terminales und der subependymalen Venen führt zur hämorrhagischen Infarzierung der weißen Substanz (Abb. 7)

2. Der Verschluss der Venae chorioideae kann zur Ventrikeleinbruchsblutung führen (Abb. 7 und 8 e)

3. Der Verschluss der Venae thalamostriatae kann zur hämorrhagischen Infarzierung der Basalganglien und der Capsula interna führen (Abb. 7).

Ein einseitiger Verschluss der tiefen venösen Drainage führt zu einseitige Läsionen (Abb. 8). Ein einseitiger Verschluss der Vena cerebri interna hat eine ipsilaterale hämorrhagische Infarzierung der betroffenen Basalganglien (Abb. 8a, b), eine entsprechende ipsilaterale Ventrikeleinbruchsblutung sowie eine einseitige hämorrhagische Infarzierung der betroffenen weißen Substanz zur Folge. Mit der farbkodierten Doppler-Sonographie kann der Verschluss einer Vena cerebri interna nachgewiesen werden (Abb. 8c), mit der gepulsten Doppler-Sonographie kann die Durchgängigkeit der kontralateralen Vene dokumentiert werden (Abb. 8d).

\section{Infarkt der A. cerebri media}

Die A. cerebri media ist die häufigste von einem Verschluss bedrohte Arterie im Kindesalter. Ähnlich wie bei Sinusvenenthrombosen spielen Thrombophilien, wie sie in Tab. 1 aufgeführt sind, eine wichtige Rolle. Weiterhin können Embolien bei Herzfehlern mit Rechts-Links-Shunt zu Verschlüssen der Arterie führen. Sonographisch imponiert eine Echogenitätsvermehrung, die dem Versorgungsgebiet der A. cerebri media entspricht (Abb. 9a). Mit der Doppler-Sonographie kann ein Verschluss oder eine hochgradige Stenose der A. cerebri media nachgewiesen werden (Abb.9b). Weiterhin lässt sich der genaue Ort der Stenose oder des Verschlusses sowie die eventuelle Rekanalisierung darstellen.

Im weiteren Verlauf kommt es bei Verschlüssen zu einer Kolliquationsnekrose mit zystischer Umwandlung des betroffenen Gebietes (Abb. 9c). Mit der farbkodierten Doppler-Sonographie können die meist mitbetroffenen Aa. thalamostriatae im Gegensatz zur gesunden Gegenseite nicht dargestellt werden (Abb.9d). Wenn kein arterieller Zufluss zu den Markabschnitten erfolgt, können die drainierenden Venae terminales und subependymales im Gegensatz zur gesunden Seite nicht dargestellt werden (Abb. 9d).

\section{AV-Malformation der Vena Galeni magna}

Die AV-Malformation der Vena Galeni magna ist die häufigste zerebrale vaskuläre Fehlbildung bei Neugeborenen. Entwicklungsgeschichtlich entstehen AV-Malformationen der Vena Galeni magna zwischen der 5. und 12. Gestationswoche durch Persistenz der mittleren prosenzephalen Vene, die den Plexus chorioideus des 3. Ventrikels drainiert.

Die Folge ist eine zunehmende Ektasie der Vena Galeni magna in der Region des Velum interpositum, in die eine Vielzahl von Arterien mündet. In erster Linie wird die AV-Malformation von den Aa. chorioideae posteriores, seltener von den Aa. thalamostriatae und Ästen der A. cerebri anterior gespeist. Durch den zunehmenden arteriovenösen Shunt kommt es zur progredienten Dilatation der AV-Malformation und zum Anstieg des zerebralen Venendruckes, der zum Hydrozephalus und zur Herzinsuffizienz führt.

Postpartal fallen die Kinder mit einer rasch progredienten Herzinsuffizienz und einem über dem Neurokranium auskultierbaren Strömungsgeräusch auf. Im zweidimensionalen Schnittbild zeigt sich eine zystische Raumforderung hinter dem 3. Ventrikel, die diesen von okzipital komprimiert (Abb.10a). Differenzialdiagnostisch müssen supratentorielle Arachnoidalzysten ausgeschlossen werden. Mit der farbkodierten Doppler-Sonographie kann der vaskuläre Ursprung der Malformation bewiesen und die zuführenden Arterien (meist Arteriae chorioideae posteriores) sowie die drainierenden Sinus (Sinus rectus und sigmoideus) dargestellt werden (Abb. 10b) $[5,10,14,15,18]$. Zuführende Arterien stellen sich farbdopplersonographisch deutlich prominenter als Arterien, die nicht in die Malformation münden, dar.

Die dopplersonographische Diagnose kann mithilfe der Kernspintomographie und MR-Angiographie bestätigt werden (Abb. 10c, d).

Mit der gepulsten Doppler-Sonographie kann in den zuführenden Arterien ein systolisch-diastolischer Vorwärtsfluss mit sehr hoher diastolischer Amplitude gefunden werden (Abb.11a). Die hohe diastolische Amplitude kommt durch den niedrigen peripheren Gefäßwiderstand zustande (Abb. 11a). Sowohl die maximalen systolischen als auch die diastolischen und mittleren 

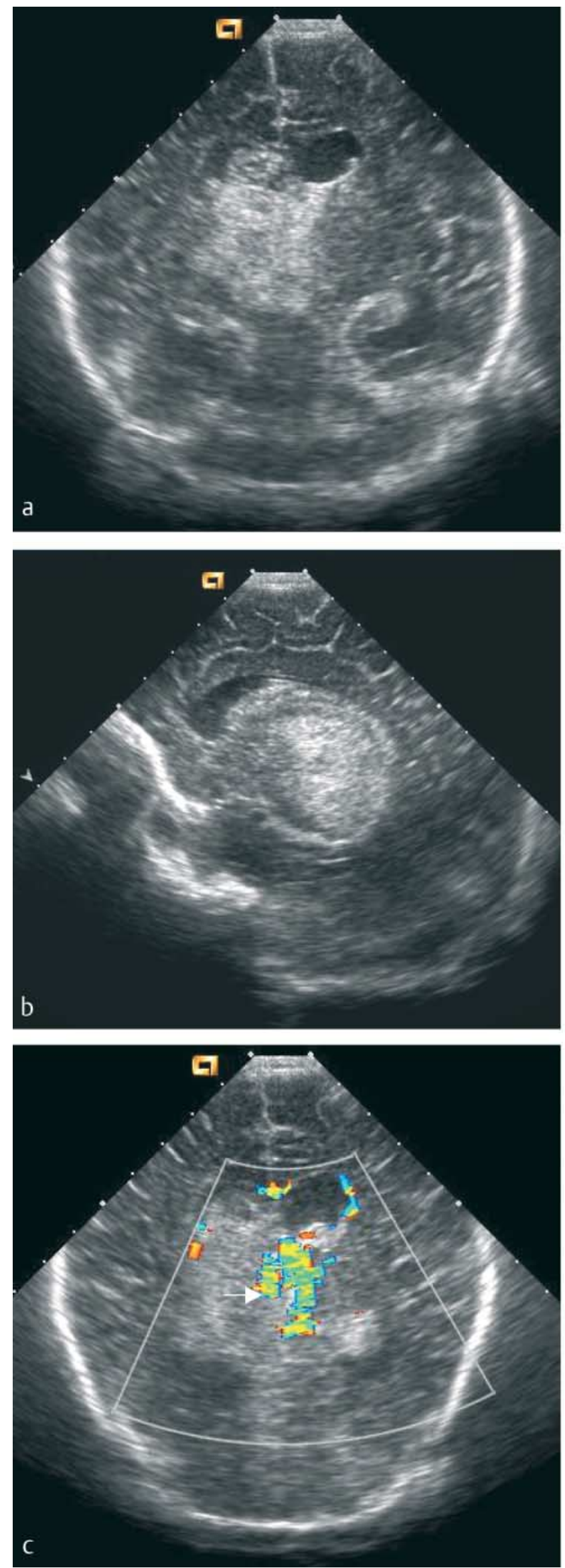
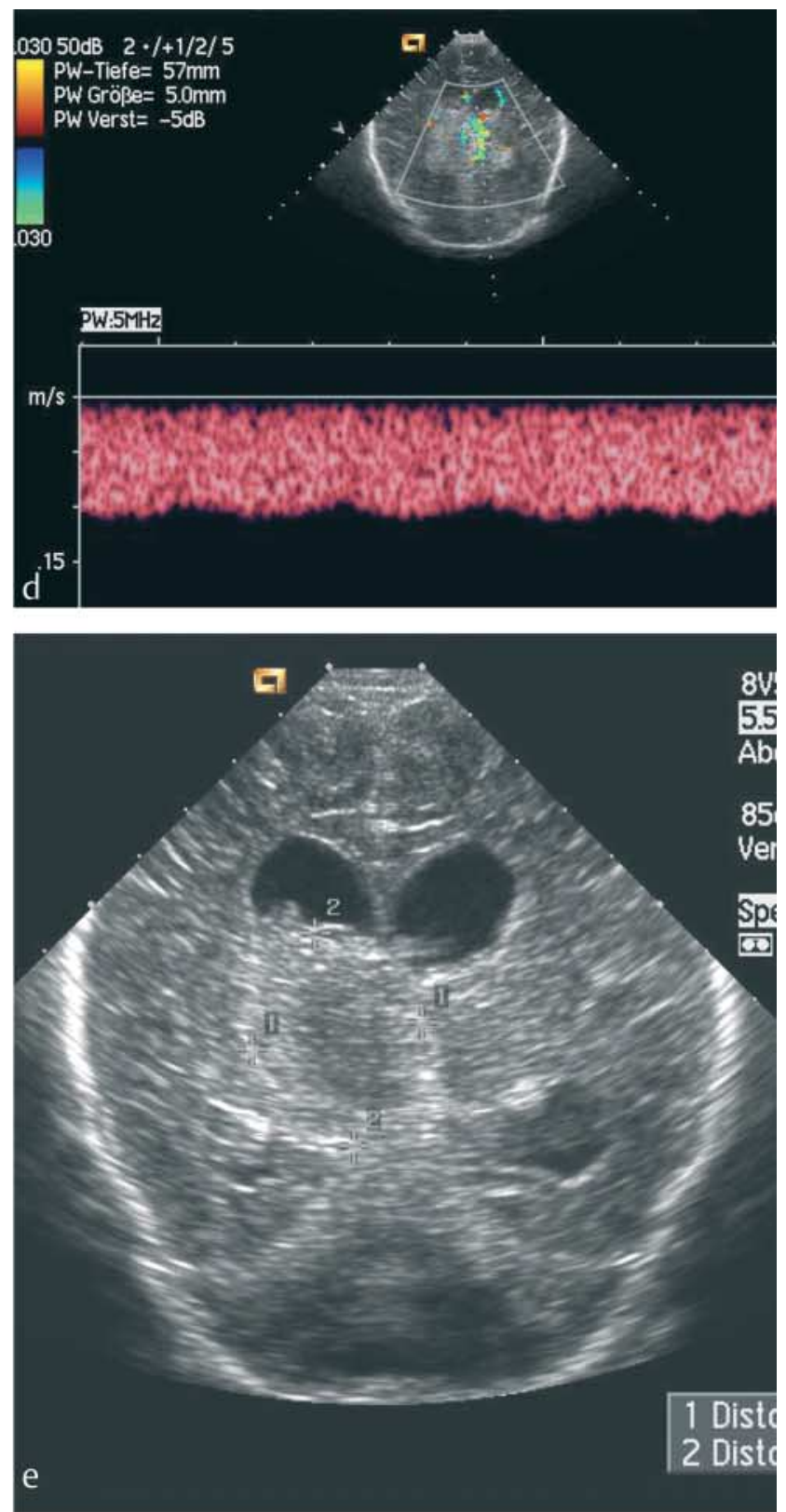

Abb. 8 a, b Komatöses Kind mit vorgewölbter Fontanelle und zerebralen Krampfanfällen. Blutung in die rechten Basalganglien und zusätzlich Ventrikeleinbruchsblutung als Hinweis auf eine tiefe Hirnvenenthrombose. a Koronarschnitt, b Sagittalschnitt. c, d Hämorrhagischer Infarkt der rechten Basalganglien als Folge eines Verschlusses der rechtsseitigen Vena cerebri interna. c Mit der farbkodierten Doppler-Sonographie ist die Vena cerebri interna auf der rechten Bildseite durchgängig, während sie auf der linken Bildseite plötzlich abbricht $(\rightarrow)$. d Normales Flussprofil in der durchgängigen Vena cerebri interna auf der rechten Bildseite. e Symmetrische Ventrikelerweiterung nach Anlage eines ventrikuloperitonealen Shunts zwei Monate nach Thrombose der rechten Vena cerebri interna. Neben einer Ventrikelerweiterung findet sich eine Echogenitätsvermehrung im Bereich der rechtsseitigen Basalganglien.

Fig. 8 a, b Comatose infant with cerebral seizures and bulging fontanelle. Hemorrhage into the right basal ganglia and intraventricular hemorrhage suspicious of deep cerebral venous thrombosis. a Coronal section, b sagittal section. c, $\mathbf{d}$ Hemorrhagic infarction of the right basal ganglia as the result of occlusion of the right internal cerebral vein. c Colour coded Doppler sonography shows patent left internal cerebral vein on the right side of the image. The internal cerebral vein on the right side of the image is patent, the vein on the left side of the image is obstructed and suddenly stops $(\rightarrow)$. d Normal flow profile and flow velocities within the patent left internal cerebral vein (right side of image). e Symmetric ventricular dilation after implantation of a ventricular peritoneal shunt 2 months after thrombosis of the right internal cerebral vein. Beside the ventricular dilation an increased echogenicity in the region of the right sided basal ganglia can be shown. 

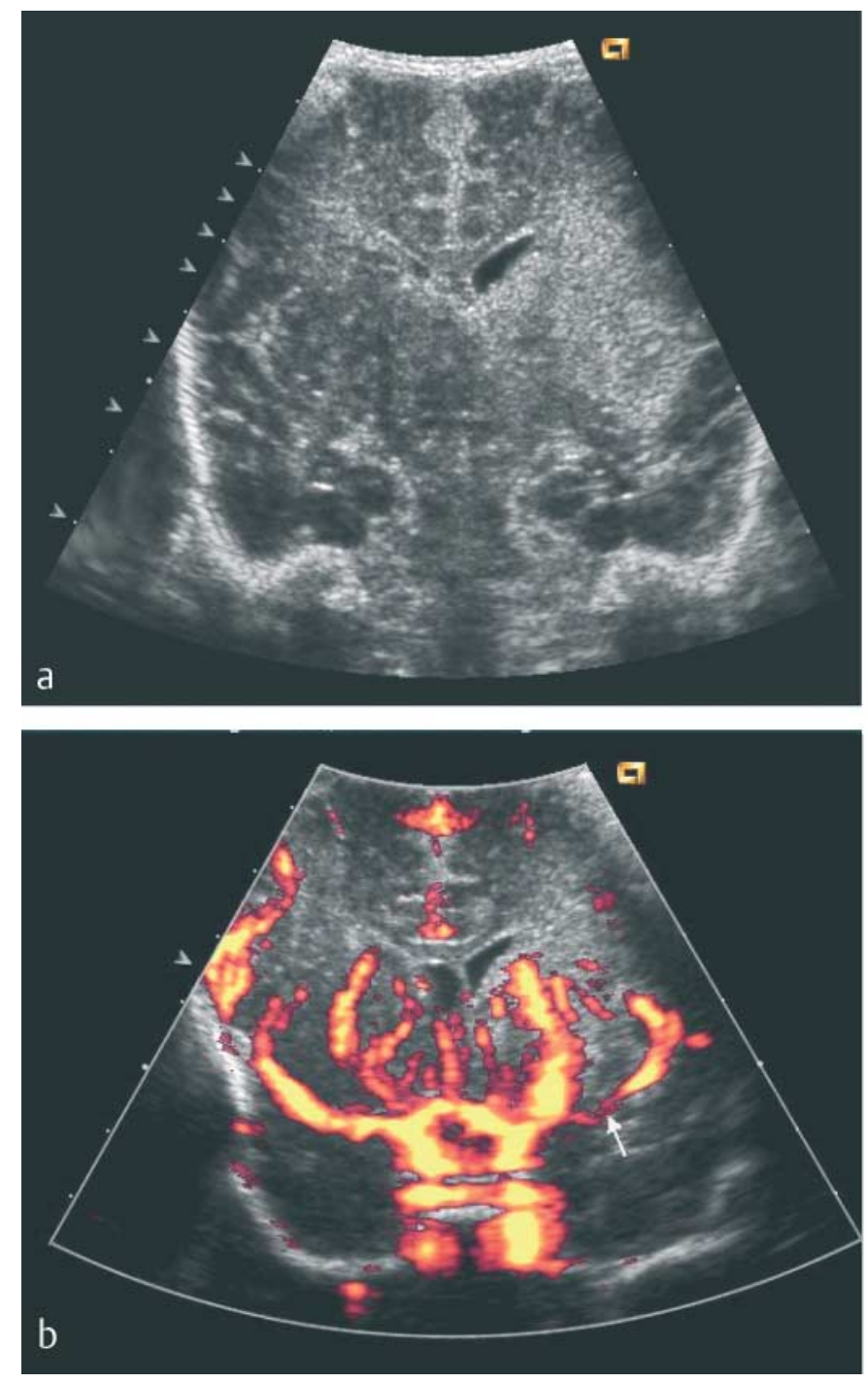

Abb. 9 a, b Infarkt der linken Arteria cerebri media. a Echogenitätsvermehrung im Versorgungsgebiet der linken $\mathrm{A}$. cerebri media auf der rechten Bildseite. b Mit dem Power-Doppler zeigt sich eine hochgradige Stenose der A. cerebri media links. Möglicherweise handelt es sich um die Rekanalisierung eines vorausgegangenen Verschlusses. c, d Zustand 6 Wochen nach Infarkt der A. cerebri media links. c Im zweidimensionalen Schnittbild zeigt sich eine multizystische Transformation des betroffenen Gebietes. d Die farbkodierte Ausschnittsvergrößerung zeigt auf der gesunden Seite eine gute arterielle Versorgung und eine gute venöse Drainage über die Venae terminales und subependymales, die auf der betroffenen Seite fehlt.

Flussgeschwindigkeiten sind in den Feeder-Arterien deutlich erhöht, was zum Abfall des Resistance-Index führt.

Mit der gepulsten Doppler-Sonographie können somit die zuführenden Arterien anhand der hohen diastolischen Amplitude erkannt werden. Demgegenüber findet man in Nichtfeeder-Arterien ein normales Flussprofil mit niedrigeren Flussgeschwindigkeiten. Um alle Feeder-Arterien zu erfassen, sollte eine komplette dopplersonographische Analyse aller Hinarterien erfolgen. Meist entspringen die Feeder-Arterien aus der A. basilaris, der A. cerebri posterior und der A. chorioidea posterior, seltener aus der A. cerebri media, thalamostriata oder A. chorioidea anterior.
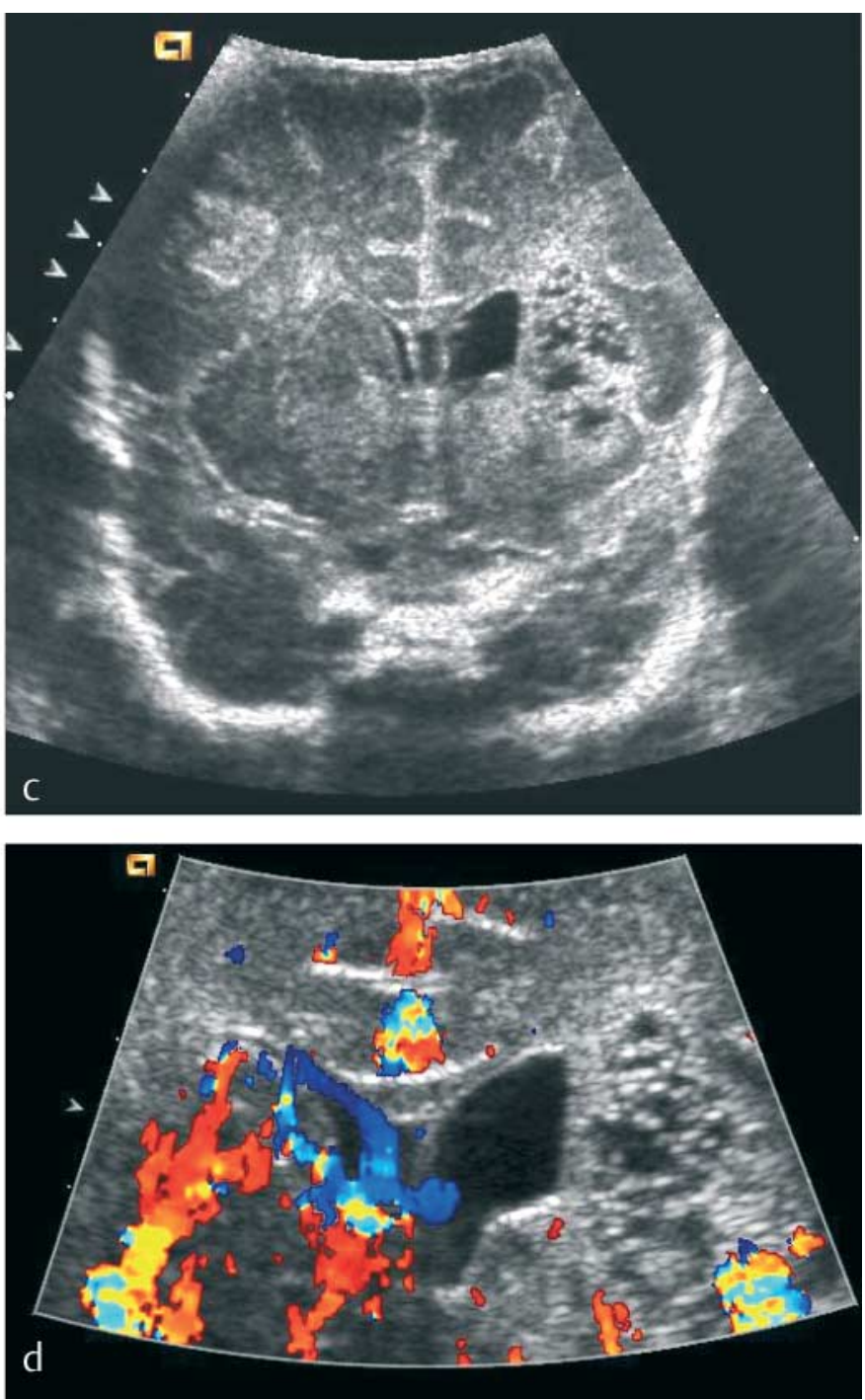

Fig. 9 a, b Infarction of the left middle cerebral artery. a Coronal scan shows an increased echogenicity in the territory of the left middle cerebral artery. b Power Doppler sonography shows severe stenosis of the left middle cerebral artery. Possibly it is the recanalisation of a preceded occlusion. c, $\mathbf{d}$ Situation 6 weeks after the infarction of the left middle cerebral artery. c 2-dimensional image shows multicystic transformation of the infarcted area. $\mathbf{d}$ Colour coded Doppler sonography shows on the non affected side a good arterial supply by the lenticulo striatic arteries and a good venous drainage by the subependymal and terminal veins. On the side of the infarction arterial inflow by the lenticulo striatic arteries as well as venous outflow by the terminal and subependymal veins is missing.

In der dilatierten AV-Malformation der Vena Galeni und im abführenden Sinus rectus findet sich spiegelbildlich ein pulsatiler Fluss mit hoher diastolischer Amplitude (Abb.11 und 12b). Die Darstellung kann sowohl im Sagittalschnitt (Abb.11b) als auch im Koronarschnitt erfolgen (Abb. 12b).

Die Doppler-Sonographie ist somit einerseits zur Diagnose der AVMalformation der Vena Galeni magna in der Lage, andererseits können die zuführenden Feeder-Arterien dargestellt und die venöse Drainage abgebildet werden [10]. Zur Sicherung der Diagnose ist die Kernspintomographie nicht erforderlich. Für die Festlegung des weiteren Procedere (palliative oder kurative Therapie) sollte jedoch die genaue Gefäßanatomie mittels Angiographie festgelegt werden. Falls man sich für ein kuratives Vorgehen entscheidet, ist 

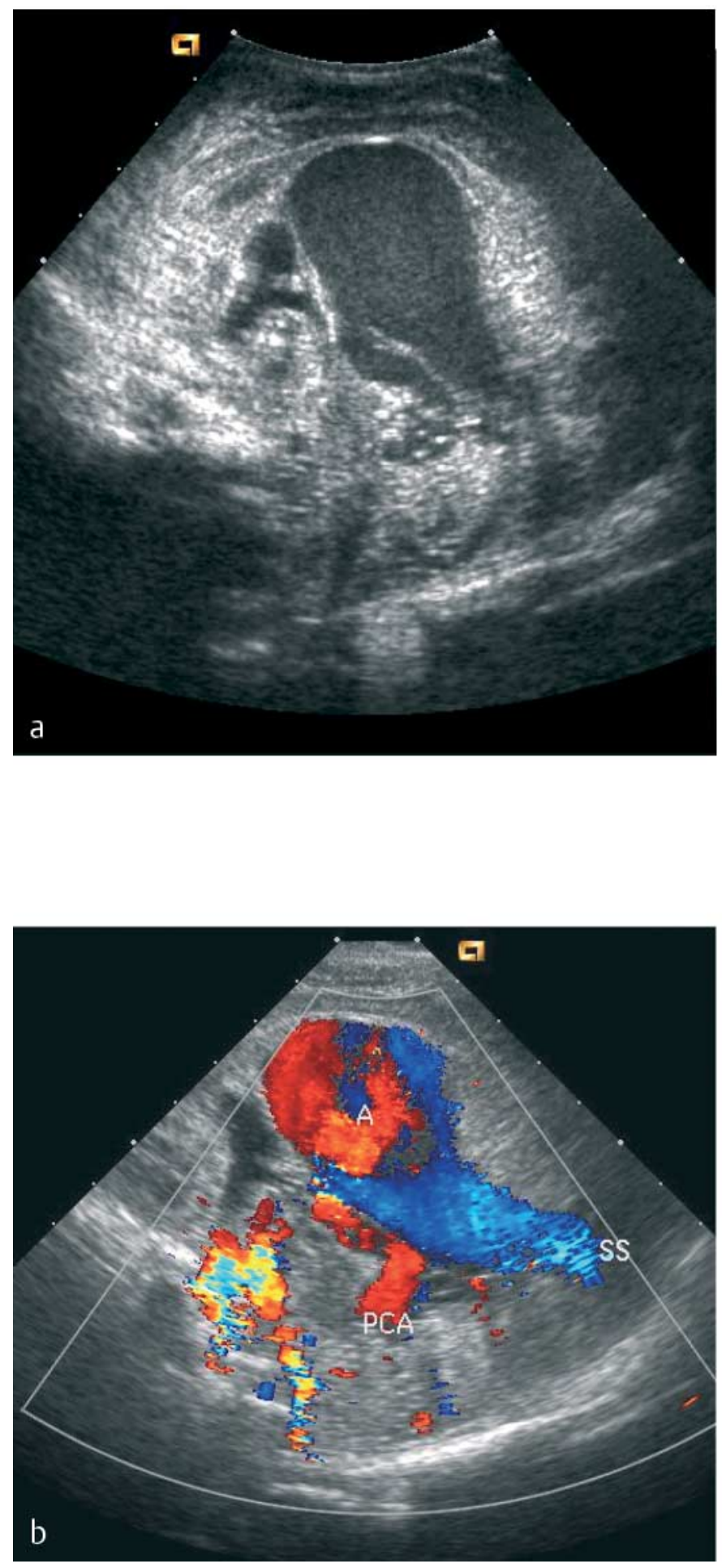

Abb. 10 AV-Malformation der Vena Galeni magna. a Im medianen Sagittalschnitt lässt sich eine zystische Raumforderung hinter dem 3. Ventrikel nachweisen, die diesen komprimiert und der AV-Malformation entspricht. b In der Raumforderung (A) kann mit der farbkodierten Doppler-Sonographie eine Blutströmung nachgewiesen werden, sodass der vaskuläre Charakter bewiesen ist. Zufluss über die Arteria cerebri posterior (PCA), Drainage über den Sinus rectus (SS). c MRI der AV-Malformation der Vena Galeni magna und d MRI-Angiographie.
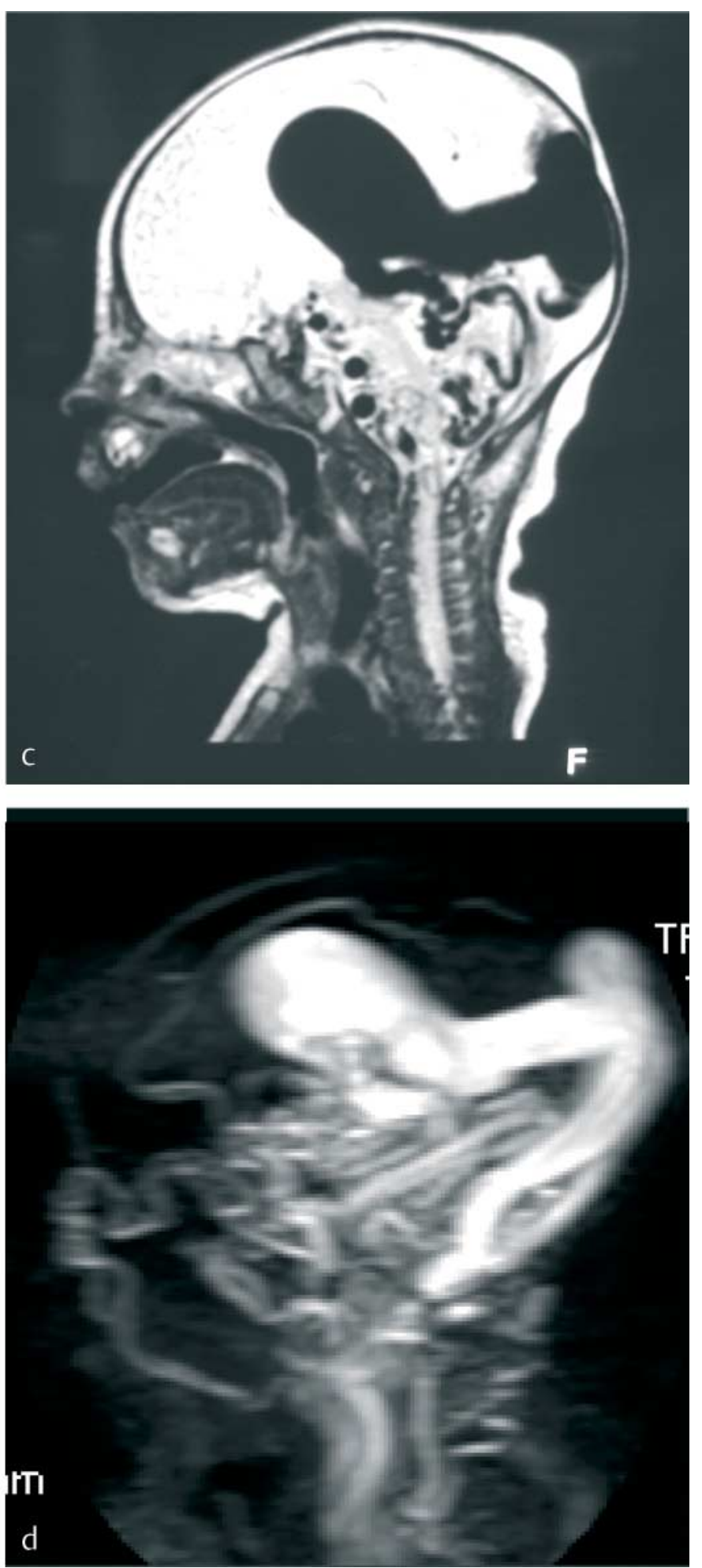

Fig. 10 AV-Malformation of the vein of Galen. a Midline sagittal section shows a cystic mass posteriorly to the $3^{\text {rd }}$ ventricle, which compresses the $3^{\text {rd }}$ ventricle and Sylvian aqueduct. The cyst is an AV-Malformation of the vein of Galen. $\mathbf{b}$ Within the cyst (A) colour coded Doppler sonography shows blood flow, demonstrating the vascular nature of the cyst. The feeder arteries originate from prominent posterior cerebral arteries (PCA). Venous drainage by the straight sinus (SS). c MRI of the AV-Malformation of the vein of Galen, $\mathbf{d}$ MRI angiogramm of the AV-Malformation. 


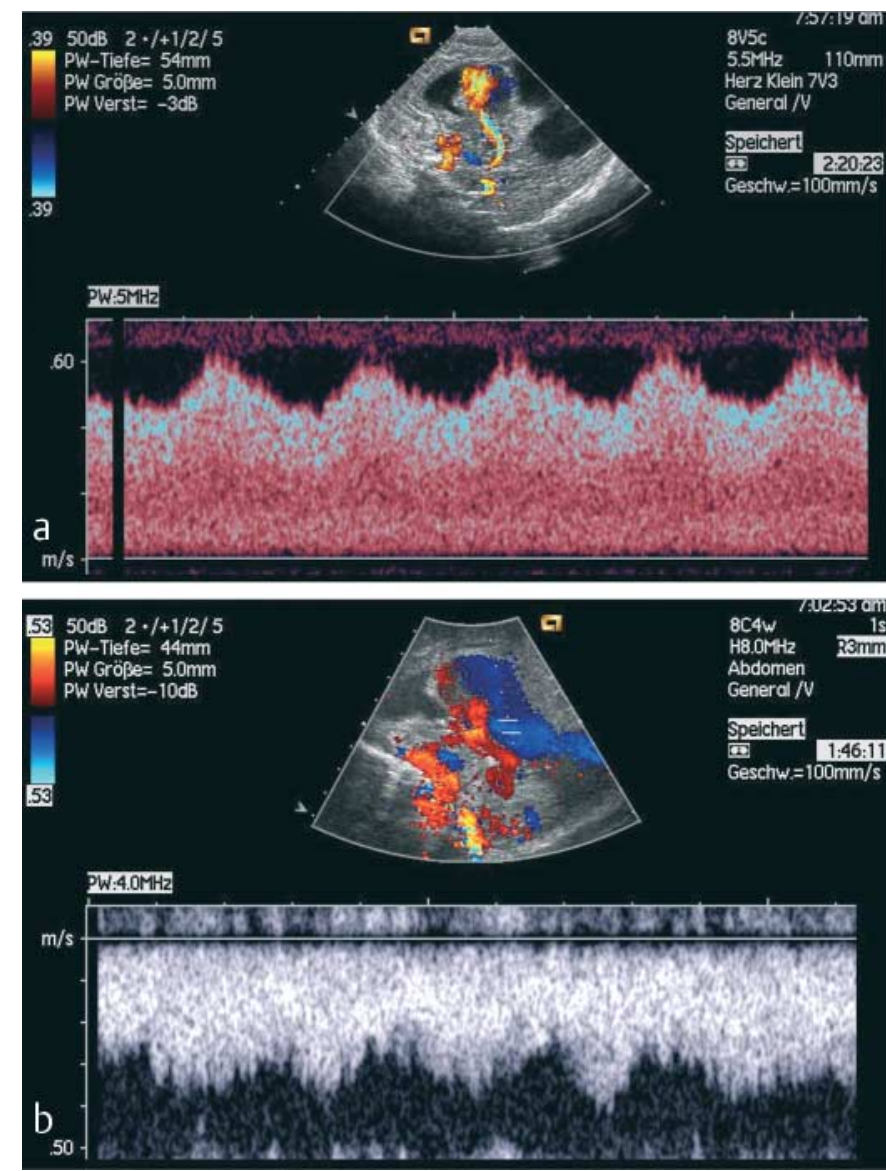

Abb. 11 AV-Malformation der Vena Galeni magna: Farbkodierte Doppler-Sonographie des arteriellen Zuflusses über die Arteria chorioidea posterior und des venösen Abflusses über die Vena Galeni magna und den Sinus rectus (Sagittalschnitt). a Arterieller Zufluss über die Arteria cerebri posterior: Hohe diastolische Amplitude als Hinweis auf den niedrigen peripheren Widerstand. b Venöse Drainage über die AVMalformation der Vena Galeni magna und den Sinus rectus: Pulsatiler venöser Fluss mit hoher diastolischer Amplitude und hohen Flussgeschwindigkeiten (Spiegelbild der arteriellen Blutströmung).

Fig. 11 AV-Malformation of the vein of Galen: Colour coded Doppler sonography of the arterial feeders by the way of the posterior choroideal arteries and of the venous drainage by the ectatic great vein of Galen and the straight sinus (sagittal section). a Arterial feeders originating from the posterior cerebral artery: High diastolic amplitude caused by the low peripheral vascular resistance. $\mathbf{b}$ Venous drainage of the AV-Malformation by the dilated straight sinus: Pulsatile venous flow with high diastolic amplitude and high flow velocities (mirror image of the flow in the feeder arteries!).

die Embolisation der AV-Malformation die Therapie der Wahl. Diese kann sowohl über den arteriellen als auch über den venösen Zugang erfolgen. Bei hämodynamisch stabilen Neugeborenen erfolgt die Embolisation im Idealfall im Alter von 5 Monaten.

Spontane Thrombosen der AV-Malformation können auftreten, sind jedoch extrem selten. Sie wurden mit einer Häufigkeit von ca. $4 \%$ beschrieben [17]. Im Falle einer spontanen Thrombose kann ein kalzifizierter Rand nachgewiesen werden [6].

Die häufigste Komplikation einer AV-Malformation der Vena Galeni magna ist ein Hydrozephalus, der bei etwa 50\% aller Kinder auftritt. Der Hydrozephalus ist in der Regel nicht durch eine Blockade des Aquädukts, sondern durch den hohen intrakraniellen Venen-
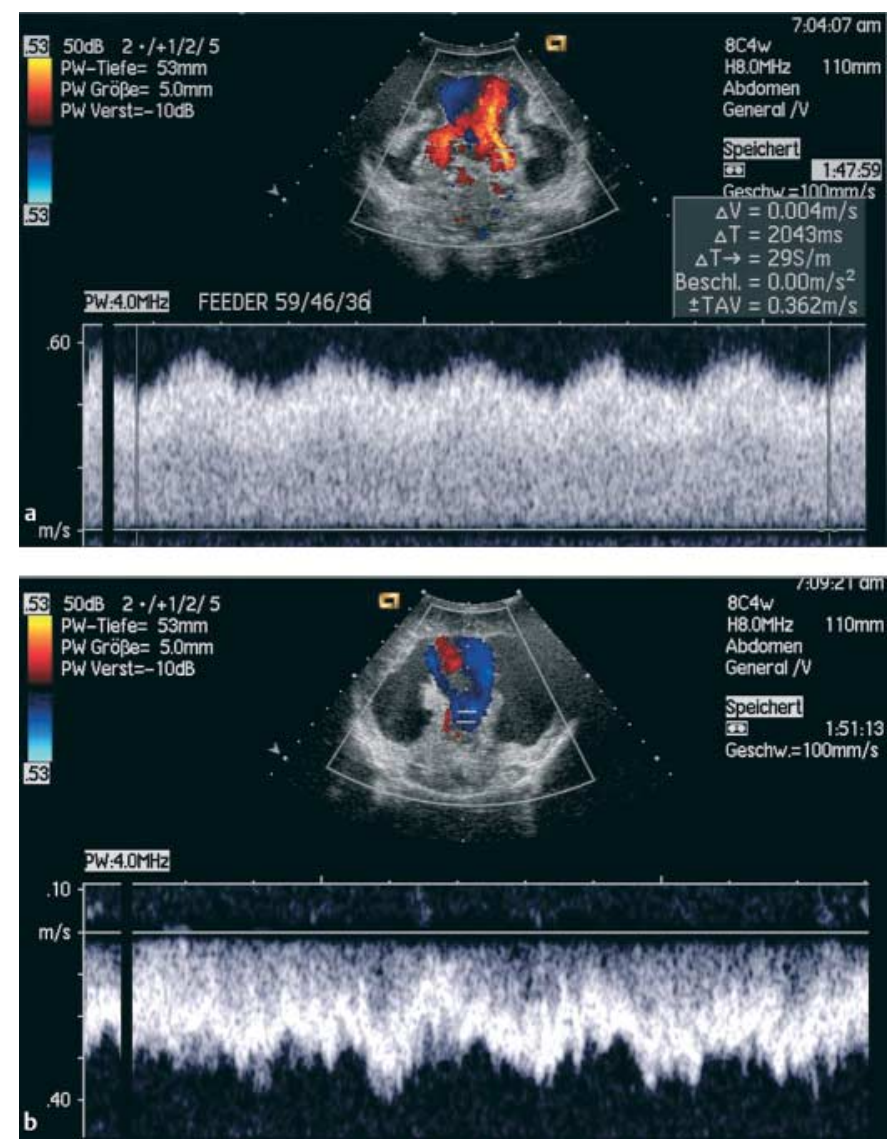

Abb. 12 AV-Malformation der Vena Galeni magna. Dopplersonographische Flussmessungen im Koronarschnitt. a Darstellung der arteriellen Feeder-Arterien (Arteriae chorioideae posteriores). Hohe diastolische Amplitude mit hohen Flussgeschwindigkeiten durch den niedrigen peripheren Gefäßwiderstand. b Venöse Drainage über die dilatierte AV-Malformation der Vena Galeni und den Sinus rectus. Das Frequenzspektum zeigt einen pulsatilen Fluss mit hoher diastolischer Amplitude (Spiegelbild des arteriellen Zuflusses).

Fig. 12 AV-Malformation of the vein of Galen. Doppler sonographic flow measurements in coronal section. a Imaging of the feeder arteries (posterior choroideal arteries). High diastolic amplitude with high flow velocities due to low peripheral vascular resistance. b Venous drainage by the ectatic vein of Galen and the straight sinus. The frequency spectrum shows pulsatile flow with a high diastolic amplitude (mirror image of the arterial flow).

druck bedingt. Teilweise konnten Venendrücke von $50 \mathrm{cmH}_{2} \mathrm{O}$ und mehr gemessen werden [20]. Dadurch wird die Resorption des Liquors in den Pacchioni-Granulationen des Subarachnoidalraums erschwert. Nach Embolisation der AV-Malformation sinkt der Venendruck und der Hydrozephalus bildet sich zurück.

Zusammenfassend ist eine genaue Kenntnis der intrakraniellen Venen für das Verständnis von hämorrhagischen Infarkten des ZNS, Sinusvenenthrombosen sowie arteriellen Verschlüssen und zerebralen vaskulären Fehlbildungen unverzichtbar. Die farbkodierte und gepulste Doppler-Sonographie ermöglicht bei offener Fontanelle die zuverlässige nichtinvasive Diagnose der wichtigsten vaskulären Erkrankungen. Weiterführende invasive Diagnostik, wie z.B. die MR-Angiographie, kann gezielt eingesetzt werden. Verlaufskontrollen können kostengünstig mit der farbkodierten Duplex-Sonographie erfolgen. 


\section{Literatur}

${ }^{1}$ Barron TF, Gusnard DA, Zimmermann RA et al. Cerebral venous thrombosis in neonates and children. Pediatr Neurol 1992; 8: 112-116

${ }^{2}$ Baumeister FA, Auberger MK, Schneider K. Thrombosis of the deep cerebral veins with excessive bilateral infarction in a premature infant with the thrombogenic $4 \mathrm{G} / 4 \mathrm{C}$ genotype of the plasminogen aktivator inhibitor-1. Eur J Pediatr 2000; 159: 239-242

${ }^{3}$ Baumeister FA, Hofer MM, Schneider K. Thrombose der V. cerebri magna. Ultraschalldiagnostik. Monatssch Kinderheilkd 2000; 148: 239-241

${ }^{4}$ Bezinque SL, Slovis TL, Touchette AS et al. Characterization of superior sagittal sinus blood flow velocity using color flow Doppler in neonates and infants. Pediatr Radiol 1997; 25: 175 - 179

${ }^{5}$ Brunelle F. Arteriovenous malformation of the vein of Galen in children. Pediatr Radiol 1997; 27: 501 - 513

${ }^{6}$ Chapman S, Hockley A. Calcification of an aneurysm of the vein of Galen. Pediatr Radiol 1989; 19: 541 -542

${ }^{7}$ Couture AP, Veyrac C. Cerebral venous thrombosis. In: Couture A, Veyrac C (Hrsg). Transfontanellar Doppler imaging in neonates. Berlin, Heidelberg, New York: Springer, 2001: 249-263

${ }^{8}$ Couture AP. Neonatal and fetal brain malformations. Aneurysm of vein of Galen. In: Couture A, Veyrac C (Hrsg). Transfontanellar Doppler imaging in neonates. Berlin, Heidelberg, New York: Springer, 2001: $282-308$

${ }^{9}$ Dean LM, Taylor GA. The intracranial venous system in infants: normal and abnormal findings on duplex and color Doppler sonography. AJR 1995; 164: 151 - 156

${ }^{10}$ Deeg KH, Scharf J. Colour doppler imaging of arteriovenous malformation of the vein of Galen in a newborn. Neuroradiology 1990; 32: $60-63$

${ }^{11}$ Deeg KH, Staudt F, Rohden Lv. Klassifikation der intrakraniellen Blutungen des Frühgeborenen. Ultraschall in Med 1999; 20: 165-170
${ }^{12}$ Deeg KH, Lode HM. Transfontanelläre Doppler-Sonographie der Hirnvenen im Säuglingsalter I - Normalbefunde. Ultraschall in Med 2005 (in Druck)

${ }^{13}$ Govaert P, Achten E, Vanhaesebrouck P et al. Deep cerebral venous thrombosis in thalamoventricular hemorrhage of the term newborn. Pediatr Radiol 1992; 22: $123-127$

${ }^{14}$ Hoffmann HJ, Chuang S, Hendrick B et al. Aneurysms of the vein of Galen. Experience at the hospital of sick children Toronto: J Neurosurg, 1982: 57: $316-322$

15 Kilic T, Pamir MN, Budd S et al. Grading and hemodynamic follow-up study of arteriovenous malformations with transcranial Doppler ultrasonography. J Ultrasound Med 1998; 17: 729-738

${ }^{16}$ Lam AH. Doppler imaging of superior sagittal sinus thrombosis. J Ultrasound Med 1995; 14: $41-46$

${ }^{17}$ Lasjaunias P. Vein of Galen aneurysmal malformation. In: Lasjaunias P (Hrsg). Vascular diseases in neonates, infants and children. Berlin, Heidelberg, New York: Springer, 1997

${ }^{18}$ Leff SL, Kronfeld G, Leonidas JC. Aneurysms of the vein of Galen. Pediatr Radiol 1989; 20: $98-100$

19 Papile L, Burstein J, Burstein R et al. Incidence and evolution of subependymal and intraventricular hemorrhage. A study of infants with birth weight less than 1500 grams. J Pediatr 1978; 92: 529- 534

${ }^{20}$ Quisling RG, Mickle PJ. Venous pressure measurements in vein of Galen aneurysms. Am J Neuroradiol 1989; 10: 411 - 417

${ }^{21}$ Taylor GA. Effect of germinal matrix hemorrhage on terminal vein position and patency. Pediatr Radiol 1995; 25: 37-40

22 Veyrac C. Germinal matrix and/or intraventricular hemorrhage in the preterm infant. In: Couture A, Veyrac C (Hrsg). Transfontanellar Doppler imaging in neonates. Berlin, Heidelberg, New York: Springer, 2001: $91-106$

${ }^{23}$ Volpe JJ. Intracranial hemorrhage: Germinal matrix -intraventricular hemorrhage of the premature infant. In: Volpe JJ (Hrsg). Neurology of the newborn. 3rd edition. Philadelphia: Saunders Williams und Wilkins, 1995: $403-463$ 